\title{
Baicalein antagonizes acute megakaryoblastic leukemia in vitro and in vivo by inducing cell cycle arrest
}

\author{
Chunjie Yu', Jiancheng Zeng ${ }^{1}$, Zhenzhen Yan ${ }^{1}, \mathrm{Zi} \mathrm{Ma}^{2}$, Shangqin Liu ${ }^{3^{*}}$ and Zan Huang ${ }^{\text {1* }}$
}

\begin{abstract}
Background: The prognosis of acute megakaryoblastic leukemia (AMKL) is really dismal, which urges for development of novel treatment. Baicalein is one type of flavonoids extracted from Scutellaria baicalensis Georgi (Huang Qin). It inhibited cell proliferation and subcutaneous tumor formation of many tumor cell lines. However, whether baicalein possesses anti-AMKL activities has not been tested.

Results: We found that baicalein potently inhibited proliferation of multiple AMKL cells including CMK, CMY, Y10, 6133, and 6133 MPL/W515L due to apoptosis and cell cycle arrest at G1 phase. Unexpectedly, caspase inhibitor z-VADfmk did not restore cell proliferation. In contrast, ectopic expression of Cyclin D1 efficiently antagonized the inhibitory effect of baicalein. In addition, baicalein induced differentiation of 6133 MPL/W515L cells. Finally, baicalein promoted mice survival and reduced disease burden in a mouse model of AMKL.
\end{abstract}

Conclusions: Baicalein possesses potent anti-AMKL activity in vitro and in vivo. Baicalein may be a potent reagent for AMKL therapy.

Keywords: AMKL, Baicalein, Proliferation, apoptosis, Cell cycle arrest, Differentiation

\section{Background}

Acute megakaryoblastic leukemia (AMKL) is a rare subtype of acute myeloid leukemia classified as M7 by FAB [1-3]. Representing approximately $1 \%$ of all leukemia during childhood, AMKL is the most common type of acute myeloid leukemia (AML) in young children with Down syndrome with an incidence of 0.5 per million per year [4]. GATA-1 mutations are tightly associated with AMKL in Down Syndrome children and sporadic mutations have found in adult AMKL $[5,6]$. The only recurrent genetic alterations identified were $O T T-M A L$ and CBFA2T3-GLIS2 in children type of AMKL [7-9]. Although intensive multidrug chemotherapy has been employed, the prognosis of AMKL is really dismal with

\footnotetext{
*Correspondence: ubeliu@aliyun.com; z-huang@whu.edu.cn

${ }^{1}$ College of Life Sciences, Wuhan University, 16 Luo-Jia-Shan Road,

Wuhan 430072, Hubei, People's Republic of China

${ }^{3}$ Department of Hematology, Zhongnan Hospital of Wuhan University,

169 Donghu Road, Wuhan 430071, Hubei, People's Republic of China
}

Full list of author information is available at the end of the article median survival time 40 weeks [10-12]. So far, no target therapy is available for AMKL. Recently, Aurora kinase A was proposed to be a therapeutic target for chemicals such as MLN8237 to promote polyploidization and differentiation in AMKL, shedding a light on target therapy of this fatal disease [13]. Nevertheless, it is still early to warrant a successful clinical result and the poor situation urges for the development of novel therapeutic methods.

Traditional Chinese herbs have been recognized as a good resource for drug development. Among them, baicalein is very attractive for its anti-inflammatory, antimicrobial, neuro-protective, and anti-cancer properties [14]. Baicalein is one type of flavonoids isolated from the dried root of Scutellaria baicalensis Georgi (Huang Qin). It has been reported to inhibit proliferation and induce apoptosis in various human cancer cell lines, such as liver, colon, breast, lung, myeloma, and pancreatic cancer cells [15-19]. Previous studies suggest baicalein and other two closely related flavonoids (wogonin and baicalin) may inhibit proliferation and induce apoptosis mainly through 
causing cell cycle arrest, modulating activities of some important signaling molecules including AKT, ІкB- $\alpha, \mathrm{p} 53$, and notch. $[18,20-22]$, promoting reactive oxygen species (ROS) product, releasing cytochrome c, regulating mitochondrial membrane potential, or activating caspase cascade [23-25]. Yet very few studies have been done in leukemic cells. Recently, wogonoside was reported to improve survival of NOD/SCID mice xenografted with AML blasts [26]. Thus these flavonoids may possess great potential for development of anti-leukemia drugs.

In the present study, we investigated the effects of baicalein on AMKL cells. We found that baicalein potently inhibited AMKL cell proliferation in vitro by inducing cell cycle arrest. In vivo, baicalein reduced disease burden and promoted mouse survival in an AMKL mouse model. Our study identified baicalein as a potent chemical compound that may be beneficial for AMKL therapy.

\section{Results}

Baicalein potently inhibits proliferation of AMKL cells

To test the effect of baicalein on AMKL cell proliferation, multiple AMKL cell lines including CMK, CMY, Y10, and 6133 were treated with baicalein and the cell proliferation was measured. We found that baicalein efficiently inhibited cell proliferation in a concentration- and timedependent manner (Fig. 1a). 6133/MPL W515L cells were derived from 6133 with MPL W515L overexpression. These cells proliferated without SCF (stem cell factor) and caused AMKL in mice [27]. Apparently, these cells retained the sensitivity to baicalein treatment similar to 6133 cells (Fig. 1a). We also tested its effect on other types of leukemic cells and observed similar results (Fig. 1b). These observations suggest that baicalein is a potent antileukemia reagent. In this study, we focused on AMKL and used 6133 and 6133/MPL W515L cells as models.

\section{Baicalein induced apoptosis in AMKL cells}

To explore how baicalein reduced AMKL cell proliferation, we measured cell death after baicalein treatment. As shown in Fig. 2a, baicalein treatment induced apoptosis evidenced by increased Annexin V staining and the cleavage of caspase 3 (Fig. 2a, b). Although caspase inhibitor Z-VAD reduced the protein level of cleaved caspase 3, Z-VAD treatment did not significantly reduce baicalein-induced apoptosis (BAI vs BAI + z-VAD) (Fig. 2c, d). Accordingly, $\mathrm{Z}$-VAD treatment failed to restore cell proliferation inhibited by baicalein (BAI vs M BAI + Z-VAD) (Fig. 2e). These results suggest that caspase activation may not be the major cause of cell proliferation inhibition by baicalein.

\section{Baicalein causes cell cycle arrest at G1/G0 phase}

To further determine whether baicalein caused cell proliferation inhibition by inducing cell cycle arrest, we first analyzed cell cycle profiles by DAPI staining in 6133 MPL W515L cells treated with baicalein. We found that baicalein treatment significantly reduced the percentage of cells at $\mathrm{S}$ phase and increased the percentage of cells at G1 phase (Fig. 3a). Consistent to cell cycle profile, baicalein treatment dramatically elevated the expression of CDK inhibitors $\mathrm{p} 21$ and $\mathrm{p} 27$ and reduced the expression of Cyclin D1 (Fig. 3b, c). We further tested whether Cyclin D1 overexpression may overcome cell proliferation inhibition by baicalein. As expected Cyclin D1 overexpression promoted cell proliferation. More importantly, Cyclin D1 overexpression efficiently rescued the cell proliferation inhibition, especially at $10 \mu \mathrm{M}$ concentration (Fig. 4a). In consistent, Cyclin D1 overexpression rescued cell cycle arrest and cell apoptosis induced by baicalein (Fig. 4b, c). These observations suggest that cell cycle arrest may play a major role in baicalein-induced cell proliferation inhibition.

\section{Baicalein induces AMKL cell differentiation and possesses anti-AMKL potential in vivo}

To test whether proliferation inhibition by baicalein was accompanied by cell differentiation, we measured CD41 expression. Baicalein treatment significantly augmented CD41 expression (Fig. 5a). 6133/MPL W515L cells have been shown to induce AMKL in mice and compounds that forced megakaryocyte differentiation possessed potent therapeutic effect on AMKL [8, 9, 13, 27]. To test the effect of baicalein on AMKL in vivo, we transplanted 6133/MPL W515L (carrying GFP for tracing) into sublethally irradiated mice and treated mice with baicalein. 2 days after transplantation, the engraftment was confirmed by monitoring the $\mathrm{GFP}^{+}$in peripheral blood from recipient mice (data not shown). Mice were then randomly divided into two groups for baicalein or vehicle treatment. We found that baicalein treatment significantly promote mice survival (Fig. 5b). Pathology analysis revealed that baicalein significantly reduced blood cell infiltration in spleen and lung (Fig. 5c). In another set of experiment, baicalein also reduced the weight of spleen compared with vehicle treated mice 2 weeks after transplantation (Fig. 5d). These results suggest that baicalein may possess anti-AMKL function.

\section{Discussion}

The prognosis of AMKL is very dismal partially due to failure to identify recurrent genetic alterations for target therapy that urges for development of novel therapeutic methods. Recently, OTT-MAL and CBFA2T3-GLIS2 have been identified as two recurrent genetic alterations in children type of AMKL and compounds forcing megakaryocyte differentiation possessed potent therapeutic effect in AMKL $[8,9,13]$. In this study, we showed that 

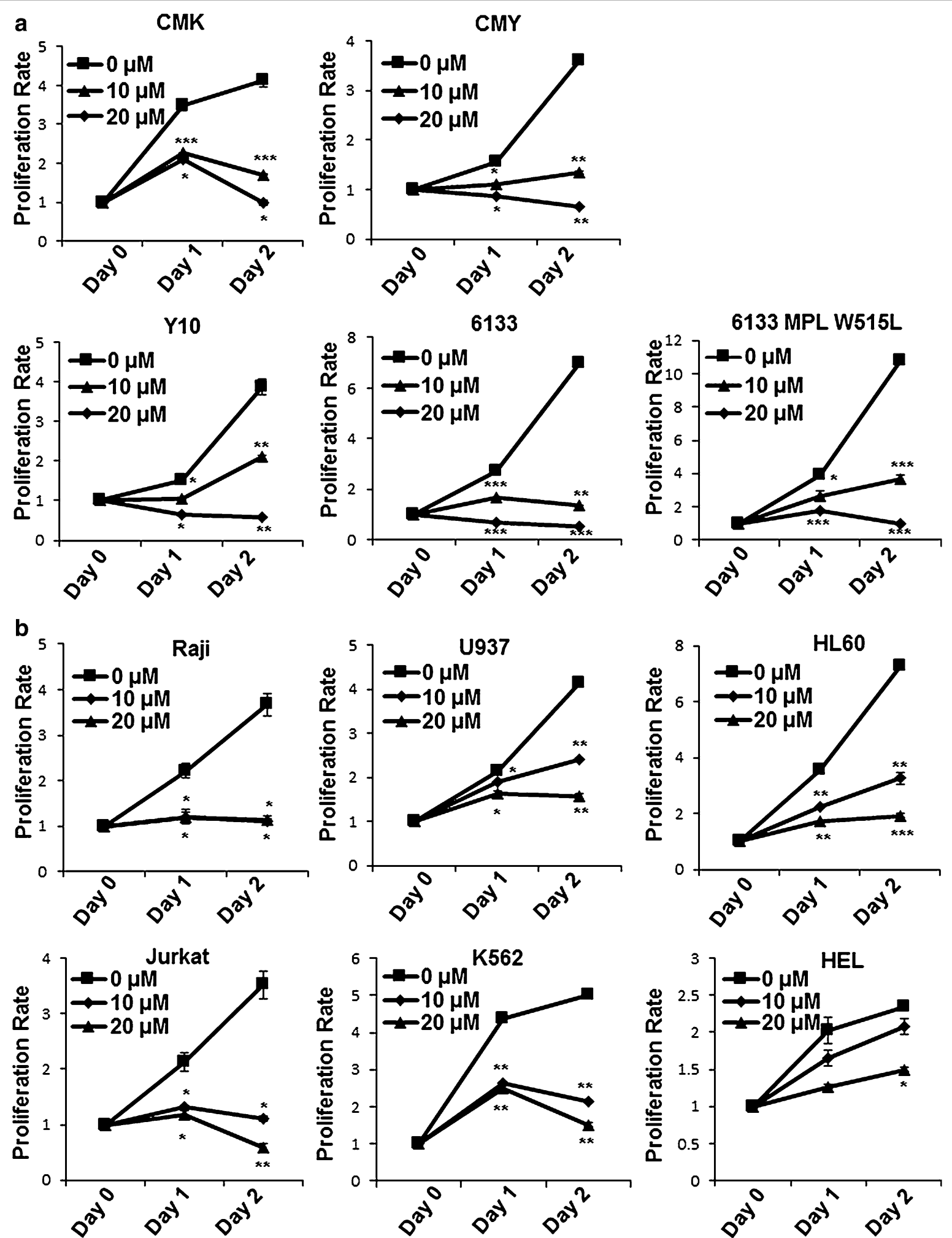

Fig. 1 Baicalein inhibited proliferation of leukemia cells. a AMKL cell lines (CMK, CMY, Y10, 6133 and 6133 MPL/W515L) and b other types of leukemic cells (Raji, U937 HL60, Jurkat and K562) were treated with or without baicalein (0, 10, and $20 \mu \mathrm{M})$. The cell numbers of viable cells were determined every $24 \mathrm{~h}$ by counting the trypan blue-excluding cells. The cell numbers were normalized to the starting cell number (day 0 ) and presented as proliferation rate. Data were statistics (Mean \pm SD) of a representative experiment (triplicates) from three independent experiments with similar results. ${ }^{*}, * *$ or ${ }^{* *}$ indicates significance $(p<0.05,0.01,0.001$, respectively) compared to vehicle treatment 


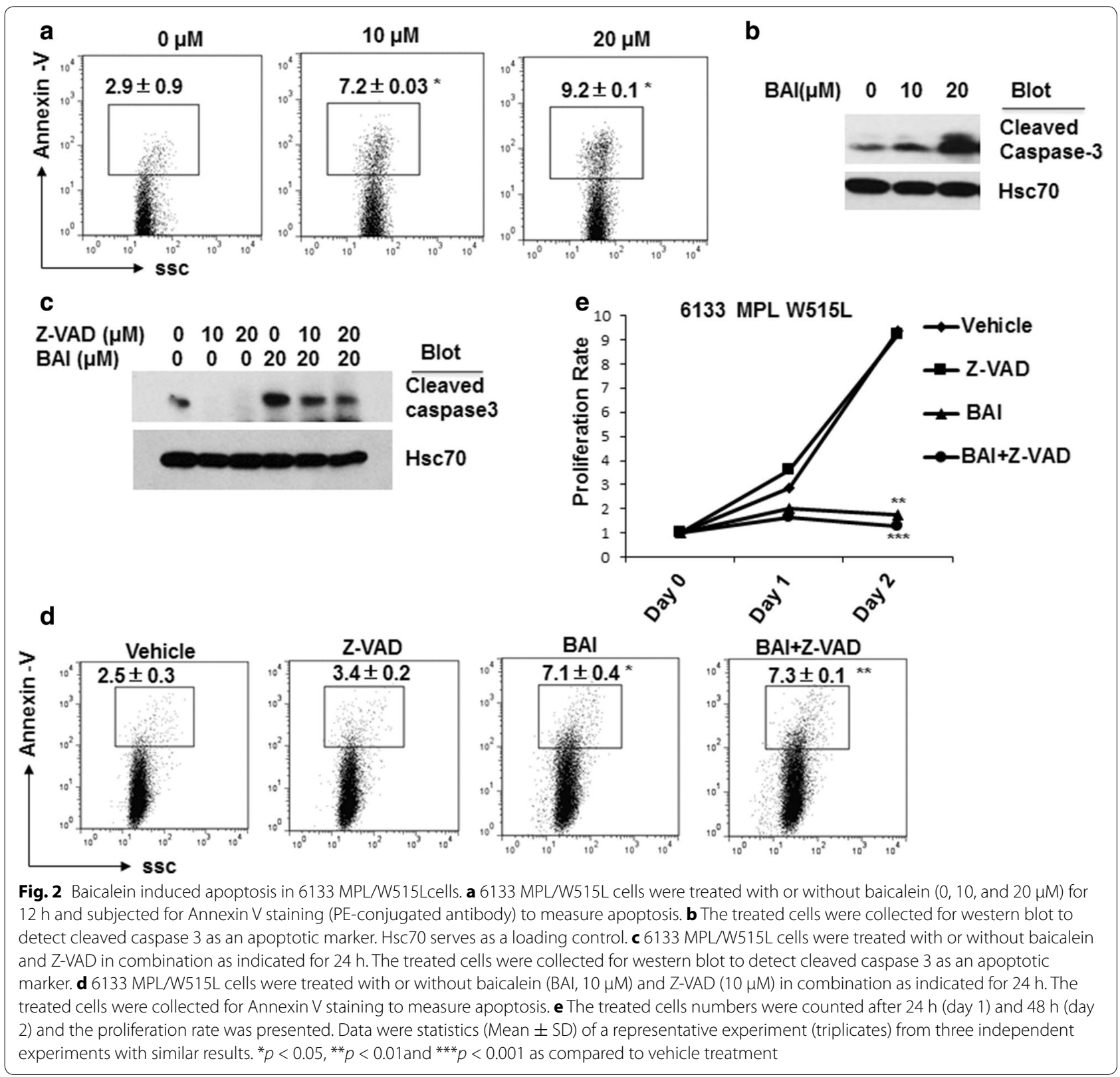

baicalein possessed anti-AMKL abilities in vitro and in vivo and might be a potent compound for developing novel AMKL therapy.

Although many previous studies have shown the antiproliferation or anti-tumor functions of baicalein or other similar bioactive components of Scutellaria such as wogonin and baicalin in many types of cancer cells, their functions in leukemia cells have not be well characterized. In one study, baicalein inhibited myeloma cell proliferation by suppressing IL-6 signaling [28]. Baicalin induced apoptosis by inducing ROS while wogonin caused apoptosis, inhibited PKC signaling, and inducing differentiation [24, 29]. In our study, we showed baicalein suppressed AMLK cell proliferation by inducing cell cycle arrest and differentiation. Although ROS seemed to be induced by baicalein, the ROS scavenger NAC (N-acetyl-L-cysteine) did not antagonize the inhibitory effect of baicalein on leukemia cell proliferation (data not shown). Considering all these observations, it is possible that baicalein may affect multiple targets and the mechanism by which baicalein exert its function depending on cell context [24, 29-31].

Other mechanism may exist for baicalein to inhibit AMKL cell proliferation. We noticed previous study 

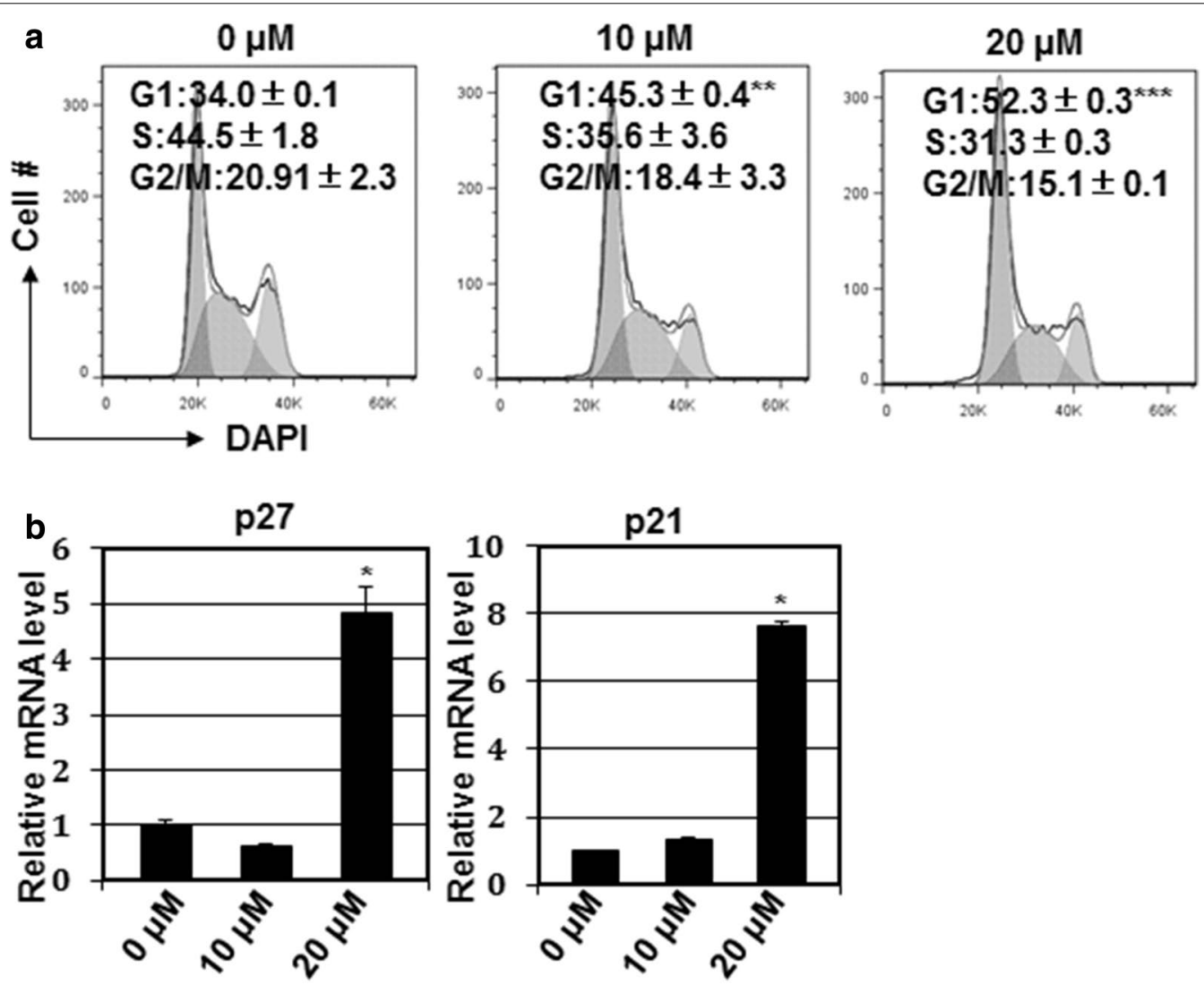

C

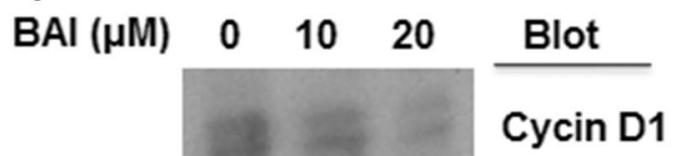

\section{$\mathrm{Hsc70}$}

Fig. 3 Baicalein caused cell cycle arrest in the G1 phase. a Cells were treated with or without baicalein $(0,10$, and $20 \mu \mathrm{M})$ for $24 \mathrm{~h}$ and collected for DAPI staining and FACS analysis. The cell cycle profile was analyzed with a Flowjo software. $\mathbf{b}, \mathbf{c}$ The treated cell were collected to measure the mRNA expression level of p21 and p27 and protein expression level of Cyclin D1. ${ }^{*} p<0.05,{ }^{* *} p<0.01$ and ${ }^{* *} p<0.001$ as compared to vehicle treatment. HSC70 was used as internal control

showing that baicalein activated Notch signaling in $\mathrm{K} 562$ cells [22]. Notch signaling was critical for early stage of megakaryocyte commitment and dysregulation of Notch signaling by OTT-MAL caused AMKL through binding to RBPJ, one downstream targets of Notch signaling. These observations suggest Notch signaling may be a common pathway for targeting in leukemia. In our model, the 6133 cells were derived from OTT-MAL knock-in mice developing AMKL. It is possible and worthy to investigate if baicalein may induce megakaryocyte differentiation and inhibit cell proliferation by modulating endogenous Notch signaling in AMKL cells.

\section{Conclusions}

Baicalein induced AMKL cells growth inhibition and apoptosis in vitro, cell cycle arrest may be the key mechanism. Baiclein can relieve disease burden in vivo. Baicalein may be a potent reagent for auxiliary AMKL therapy.

\section{Methods}

Cell culture and chemical compounds

CMK, CMY, Y10, 6133 MPL/W515L (AMKL cell lines), Raji (human lymphoma cell line), U937 (human macrophage cell line), HL60 (human promyelocytic leukemia 


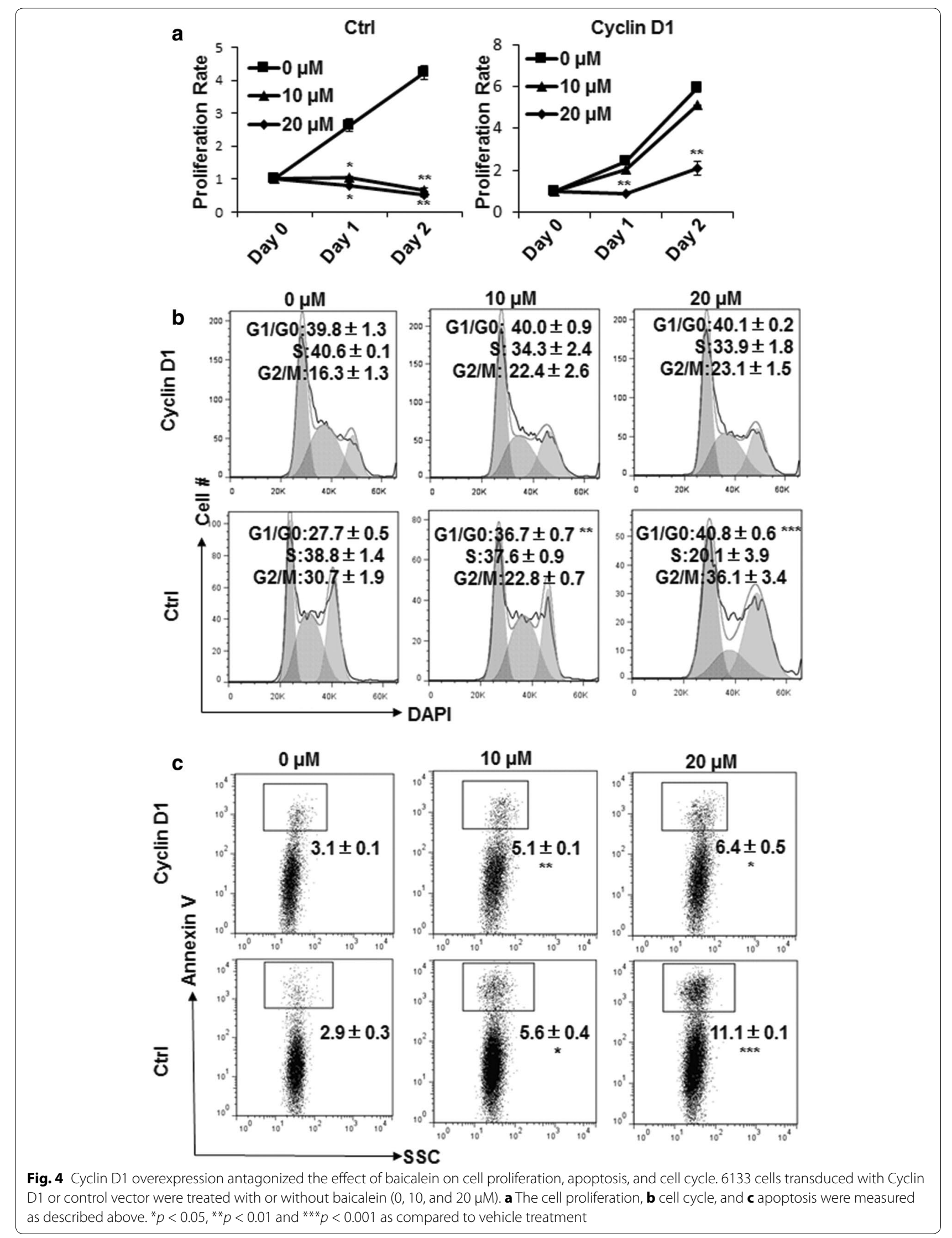




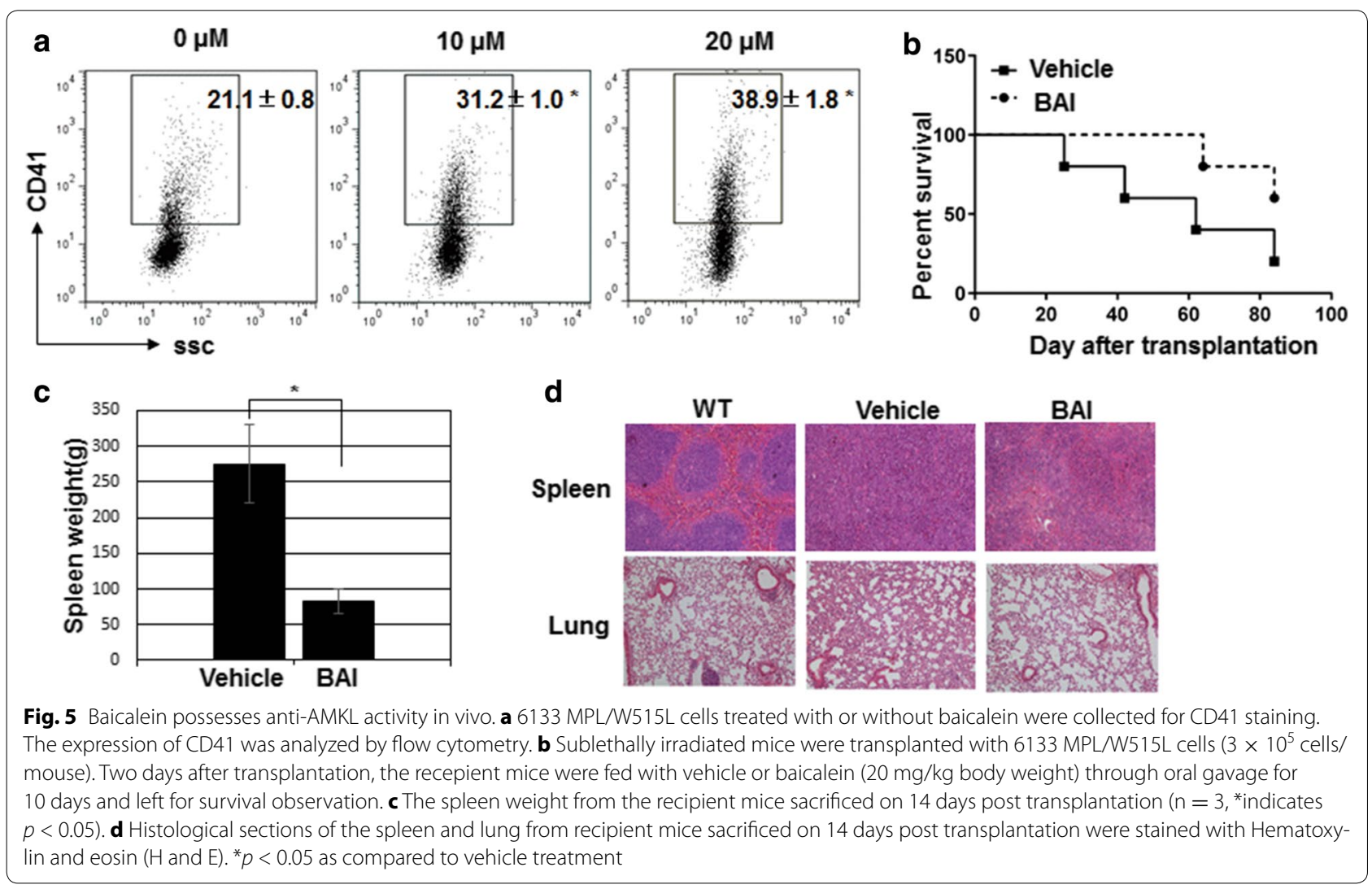

cells), Jurkat (acute T cell leukemia), K562 (chronic myelogenous leukmia), and HEL (human erythroleukemia cell) cells were cultured in RPMI-1640 media containing $10 \%$ fetal bovine serum (FBS) and $1 \%$ penicillin-streptomycin (Invitrogen, Carlsbad, CA, USA). 6133 cells were cultured in RPMI-1640 media supplemented with $1 \%$ KIT Ligand conditioned medium as previously described [32]. Baicalein was purchased from Shanxi Ciyuan Biotech Co., Ltd (Xi'an, China). z-VAD-fmk was purchased from EMD Millipore (Billerica, MT, USA). DAPI and NAC (N-acetyl-L-cysteine) were purchased from Sigma (St. Louis, MO, USA).

\section{Cell proliferation assay}

Cells $\left(5 \times 10^{5}\right.$ cells/well) were seeded in a 12 -well plate. Drugs were added to the medium at the various concentrations as indicated. After 24 and $48 \mathrm{~h}$ incubation, cells were stained with Trypan blue and the Trypan blueexcluded live cells were counted under a light microscopy. The cell numbers were normalized to the starting cell number and presented as proliferation rate. All cell proliferation curves were statistics of one representative experiment (triplicates) from three independent experiments with similar results.

\section{Cell cycle profiling}

Cell cycle profile was assayed by staining with DAPI. Briefly, baicalein-treated cells were fixed, permeablized, and stained with $10 \mu \mathrm{g} / \mathrm{ml}$ DAPI. Data were acquired through Flow Cytometry and the cell cycle profile was analyzed by a FlowJo software (Tree Star,). Flow cytometry was performed on a Calibur (BD Biosciences, Franklin Lakes, NJ, USA) and data were analyzed by a FlowJo software.

\section{Western blot analysis}

Western blot analysis was performed as previously described [32]. Membranes were blotted with antibodies detecting cleaved caspase-3, Cyclin D1, and HSC70. Cleaved caspase- 3 and Cyclin D1 antibodies were purchased from Cell Signaling Technologies. HSC70 and horseradish peroxidase-conjugated secondary antibody were purchased from Santa Cruz Biotechnology (Santa Cruz, CA). DAPI and Annexin-V flow antibody were purchased from BD.

\section{Quantitative RT-PCR}

The quantitative RT-PCR was performed as previously described [33]. The PCR primer sets used to detect p21 and p27 were as following: 
p21: sense 5-ACCAGCCTGACAGATTTCTA-3 and antisense 5-TGACCCACAGCAGAAGAG-3; P27: sense 5-AGTGTCCAGGGATGAGGA-3 and antisense 5-GG GAACCGTCTGAAACAT-3. The internal control using the primers were: GAPDH: sense 5-GGTGAAGGTCG GTGTGAACG-3 and antisense 5-CTCGCTCCTGGA AGATGGTG-3.

\section{Retroviral transduction}

For Cyclin D1 overexpression, retrovirus (pMIGR1) was used. The retroviral stocks were prepared as previously described and used for infection of 6133 cells [32]. We obtain 6133 cells with stable expression of Cyclin D1 by flow cytometry sorting GFP + cells.

\section{AMKL mouse model}

AMKL mouse model was established as previously described [13]. Briefly, recipient mice were sublethally $(600 \mathrm{rad})$ irradiated and 6133/MPL W515L cells $\left(3 \times 10^{5}\right)$ were introduced into recipient mice through tail vein injection. Two days after injection, mice were randomly divided into control and experiment groups and received vehicle or baicalein ( $20 \mathrm{mg} / \mathrm{kg}$ body weight) through oral gavage for 10 days. Mice were sacrificed on day 14 for disease burden analysis or maintained to end point for survival rate analysis. Log-rank (Mantel-Cox) test was performed to measure the statistic significance of the difference between vehicle and baicalein treatment groups. All procedures involving animals were approved by the Animal Care and Use Committee of Wuhan University.

\section{Statistical analysis}

Student's $t$-test (unpaired, two-tail) was used for statistical analysis and a $p$ value $<0.05$ was considered as significance.

\begin{abstract}
Abbreviations
AMKL: acute megakaryoblastic leukemia; SCF: stem cell factor; NAC: N-acetyl-Lcysteine; ROS: reactive oxygen species; DCFH-DA: 2', 7'-dihydrodichlorofluorescein diacetate; FACS: fluorescence activated cell sorter; DAPI: 4', 6-diamidino-2-phenylindole; GAPDH: glyceraldehyde 3-phophate; HSC70: heat Shock Cognate Protein 70.
\end{abstract}

\section{Authors' contributions}

CY, JZ and ZY performed experiments; CY and JZ designed experiments and analyzed data; $C Y$ and ZH wrote the manuscript; ZM, SL and ZH supervised the project. All authors read and approved the final manuscript.

\footnotetext{
Author details

${ }^{1}$ College of Life Sciences, Wuhan University, 16 Luo-Jia-Shan Road, Wuhan 430072, Hubei, People's Republic of China. ${ }^{2}$ Department of Chinese Medicine, Zhongnan Hospital of Wuhan University, Wuhan, Hubei, People's Republic of China. ${ }^{3}$ Department of Hematology, Zhongnan Hospital of Wuhan University, 169 Donghu Road, Wuhan 430071, Hubei, People's Republic of China.
}

\section{Acknowledgements}

This study was supported by National Nature Science Foundation (31371481 to Z. Huang; 81272627, 81470007 to S. Liu), And Program for New Century Excellent Talents in University (NCET) of Ministry of Education of China (Grant No. NCET-12-0422 to Z. Huang).

\section{Competing interests}

The authors declare that they have no competing interests.

Received: 14 January 2016 Accepted: 18 February 2016

Published online: 01 April 2016

\section{References}

1. Roy A, Roberts I, Norton A, Vyas P. Acute megakaryoblastic leukaemia (AMKL) and transient myeloproliferative disorder (TMD) in Down syndrome: a multistep model of myeloid leukaemogenesis. Br J Haematol. 2009;147:3-12.

2. Bidwai-Bhattacharjee M, Shome DK, Srinivas M, Ghosh K, Verma S, Mohanty D, et al. Acute megakaryoblastic leukaemia-an underdiagnosed entity. Indian J Cancer. 1986;23:75-82.

3. Cairney AE, McKenna R, Arthur DC, Nesbit ME Jr, Woods WG. Acute megakaryoblastic leukaemia in children. Br J Haematol. 1986;63:541-54.

4. Hama A, Yagasaki H, Takahashi Y, Nishio N, Muramatsu H, Yoshida N, et al. Acute megakaryoblastic leukaemia (AMKL) in children: a comparison of AMKL with and without Down syndrome. Br J Haematol. 2008;140:552-61.

5. Hitzler JK, Cheung J, Li Y, Scherer SW, Zipursky A. GATA1 mutations in transient leukemia and acute megakaryoblastic leukemia of Down syndrome. Blood. 2003;101:4301-4.

6. Harigae H, Xu G, Sugawara T, Ishikawa I, Toki T, Ito E. The GATA1 mutation in an adult patient with acute megakaryoblastic leukemia not accompanying Down syndrome. Blood. 2004;103:3242-3.

7. Mercher T, Busson-Le Coniat M, Nguyen Khac F, Ballerini P, Mauchauffe $\mathrm{M}$, Bui $\mathrm{H}$, et al. Recurrence of OTT-MAL fusion in $\mathrm{t}(1 ; 22)$ of infant AML-M7 Genes Chromosom Cancer. 2002;33:22-8.

8. Thiollier C, Lopez CK, Gerby B, Ignacimouttou C, Poglio S, Duffourd Y, et al. Characterization of novel genomic alterations and therapeutic approaches using acute megakaryoblastic leukemia xenograft models. J Exp Med. 2012;209:2017-31.

9. Gruber TA, Larson Gedman A, Zhang J, Koss CS, Marada S, Ta HQ, et al. An Inv(16)(p13.3q24.3)-encoded CBFA2T3-GLIS2 fusion protein defines an aggressive subtype of pediatric acute megakaryoblastic leukemia. Cancer Cell. 2012;22:683-97.

10. Sharma S, Nangia A, Jain Malhotra S, Narayan S, Harbhajanka A, Singh S. Clinico-haematological profile of acute megakaryoblastic leukaemia: report of five cases. Adv Hematol. 2009;2009:461912.

11. Al-Ahmari A, Shah N, Sung L, Zipursky A, Hitzler J. Long-term results of an ultra low-dose cytarabine-based regimen for the treatment of acute megakaryoblastic leukaemia in children with Down syndrome. $\mathrm{Br} J$ Haematol. 2006;133:646-8.

12. Ninomiya H, Nakazawa M, Shibuya A, Aoki Y, Nagasawa T, Abe T. Successful treatment of acute megakaryoblastic leukaemia. Scand J Haematol. 1986;36:147-53.

13. Wen Q, Goldenson B, Silver SJ, Schenone M, Dancik V, Huang Z, et al. Identification of regulators of polyploidization presents therapeutic targets for treatment of AMKL. Cell. 2012;150:575-89.

14. Li-Weber M. New therapeutic aspects of flavones: the anticancer properties of Scutellaria and its main active constituents Wogonin, Baicalein and Baicalin. Cancer Treat Rev. 2009;35:57-68.

15. Kim DH, Hossain MA, Kang YJ, Jang JY, Lee YJ, Im E, et al. Baicalein, an active component of Scutellaria baicalensis Georgi, induces apoptosis in human colon cancer cells and prevents AOM/DSS-induced colon cancer in mice. Int J Oncol. 2013;43(5):1652-8.

16. Lee JH, Li YC, Ip SW, Hsu SC, Chang NW, Tang NY, et al. The role of Ca2+ in baicalein-induced apoptosis in human breast MDA-MB-231 cancer cells through mitochondria- and caspase-3-dependent pathway. Anticancer Res. 2008;28:1701-11. 
17. Lee HZ, Leung HW, Lai MY, Wu CH. Baicalein induced cell cycle arrest and apoptosis in human lung squamous carcinoma $\mathrm{CH} 27$ cells. Anticancer Res. 2005;25:959-64.

18. Ma Z, Otsuyama K, Liu S, Abroun S, Ishikawa H, Tsuyama N, et al. Baicalein, a component of Scutellaria radix from Huang-Lian-Jie-Du-Tang (HLJDT), leads to suppression of proliferation and induction of apoptosis in human myeloma cells. Blood. 2005;105:3312-8.

19. Takahashi H, Chen MC, Pham H, Angst E, King JC, Park J, et al. Baicalein, a component of Scutellaria baicalensis, induces apoptosis by $\mathrm{Mcl}-1$ down-regulation in human pancreatic cancer cells. Biochim Biophys Acta. 2011;1813:1465-74

20. Chao Jl, Su WC, Liu HF. Baicalein induces cancer cell death and proliferation retardation by the inhibition of CDC2 kinase and survivin associated with opposite role of p38 mitogen-activated protein kinase and AKT. Mol Cancer Ther. 2007:6:3039-48.

21. Lee DH, Kim C, Zhang L, Lee YJ. Role of p53, PUMA, and Bax in wogonininduced apoptosis in human cancer cells. Biochem Pharmacol. 2008;75:2020-33.

22. Wang AM, Ku HH, Liang YC, Chen YC, Hwu YM, Yeh TS. The autonomous notch signal pathway is activated by baicalin and baicalein but is suppressed by niclosamide in K562 cells. J Cell Biochem. 2009;106:682-92.

23. Ling Y, Chen Y, Chen P, Hui H, Song X, Lu Z, et al. Baicalein potently suppresses angiogenesis induced by vascular endothelial growth factor through the $\mathrm{p} 53 / \mathrm{Rb}$ signaling pathway leading to G1/S cell cycle arrest. Exp Biol Med (Maywood). 2011;236:851-8.

24. Lu HF, Hsueh SC, Ho YT, Kao MC, Yang JS, Chiu TH, et al. ROS mediates baicalin-induced apoptosis in human promyelocytic leukemia HL-60 cells through the expression of the Gadd153 and mitochondrial-dependent pathway. Anticancer Res. 2007;27:117-25.

25. Yu JQ, Liu HB, Tian DZ, Liu YW, Lei JC, Zou GL. Changes in mitochondrial membrane potential and reactive oxygen species during wogonin-induced cell death in human hepatoma cells. Hepatol Res 2007;37:68-76.

26. Chen Y, Hui H, Yang H, Zhao K, Qin Y, Gu C, et al. Wogonoside induces cell cycle arrest and differentiation by affecting expression and subcellular localization of PLSCR1 in AML cells. Blood. 2013;121:3682-91.

27. Mercher T, Raffel GD, Moore SA, Cornejo MG, Baudry-Bluteau D, Cagnard $\mathrm{N}$, et al. The OTT-MAL fusion oncogene activates RBPJ-mediated transcription and induces acute megakaryoblastic leukemia in a knockin mouse model. J Clin Investig. 2009;119:852-64.

28. Liu S, Ma Z, Cai H, Li Q, Rong W, Kawano M. Inhibitory effect of baicalein on IL-6-mediated signaling cascades in human myeloma cells. Eur J Haematol. 2010;84:137-44.

29. Zhang HW, Yang Y, Zhang K, Qiang L, Yang L, Yang L, et al. Wogonin induced differentiation and G1 phase arrest of human U-937 leukemia cells via PKCdelta phosphorylation. Eur J Pharmacol. 2008;591:7-12

30. Shieh DE, Cheng HY, Yen MH, Chiang LC, Lin CC. Baicalin-induced apoptosis is mediated by $\mathrm{Bcl}$-2-dependent, but not p53-dependent, pathway in human leukemia cell lines. Am J Chin Med. 2006;34:245-61.

31. Lee WR, Shen SC, Lin HY, Hou WC, Yang LL, Chen YC. Wogonin and fisetin induce apoptosis in human promyeloleukemic cells, accompanied by a decrease of reactive oxygen species, and activation of caspase 3 and $\mathrm{Ca}(2+)$-dependent endonuclease. Biochem Pharmacol. 2002;63:225-36.

32. Huang Z, Richmond TD, Muntean AG, Barber DL, Weiss MJ, Crispino JD. STAT1 promotes megakaryopoiesis downstream of GATA-1 in mice. J Clin Investig. 2007;117:3890-9.

33. Liu L, Wen Q, Gong R, Gilles L, Stankiewicz MJ, Li W, et al. PSTPIP2 dysregulation contributes to aberrant terminal differentiation in GATA-1-deficient megakaryocytes by activating LYN. Cell Death Dis. 2014;5:e988.

\section{Submit your next manuscript to BioMed Central and we will help you at every step:}

- We accept pre-submission inquiries

- Our selector tool helps you to find the most relevant journal

- We provide round the clock customer support

- Convenient online submission

- Thorough peer review

- Inclusion in PubMed and all major indexing services

- Maximum visibility for your research

Submit your manuscript at www.biomedcentral.com/submit

\section{(O) Biomed Central}

